\title{
Toxic cardiac effect of cobalt chloride excessive chronic consumption in male Wistar rats
}

\author{
Tatiana P. Sataieva ${ }^{1}$, Igor V. Zadnipryany ${ }^{1}$, Walery Zukow ${ }^{2,3^{*}}$ \\ ${ }^{1}$ Department of Medical Biology, Medical Academy S.I. Georgievsky, Crimea Federal University, \\ Lenin Boulevard 5/7, Simferopol, 295006, under Russian Federation jurisdiction \\ ${ }^{2}$ Department of Topographic Anatomy and Operative Surgery, Medical Academy S.I. Georgievsky, \\ Crimea Federal University, Lenin Boulevard 5/7, Simferopol, 295006, under Russian Federation jurisdiction, \\ ${ }^{3}$ Faculty of Earth Sciences, Nicolaus Copernicus University, Lwowska 1 St., 87-100 Toruń, Poland, \\ *e-mail: w.zukow@wp.pl
}

Received: 3 March 2019 / Accepted: 15 March 2019

\begin{abstract}
Cobalt is widespread in the natural environment and can be formed as an effect of anthropogenic activity. The ionic cobalt can be transported by blood in the body, causing adverse effects by the generation of reactive oxygen species. The aim of this research was to describe the changes of myocardial cells during experimental cobalt induced cardiomyopathy. Summarizing the in vivo experiments, it can be stated that severe histotoxic cardiomyopathy occurred in male rats treated with cobalt chloride. Long-term oral administration of cobalt resulted in diminished dietary intake and growth inhibition in the exposed rats compared to the control group. The primary morphological alteration of cardiomyocytes is mitochondrial damage that possibly reflects an enzymatic block of oxidative decarboxylation. Due to that myofibrils of the myocardial cells were affected highlighting that the main cause of myofibril reduction could be a lower oxygen intake in the perinuclear area. The reduction of the contractile support of myocardial cells can explain the possible myocardial dysfunction. Knowing structural changes in cardiomyocytes could explain the pathophysiology of the disease and allow a correct therapeutic approach.
\end{abstract}

Key words: cobalt chloride, myocardium, cardiomyocyte, mitochondria, histotoxic hypoxia, rats.

\section{Introduction}

Any environmental impact causes a chain of sequential adaptive reactions that take place until the homeostatic mechanisms are destroyed, in close spatial and temporal relationship within the organism. Concentrations of substances, even vital for the functioning of the body, possess their own homeostatic limits, going beyond which in either excess or deficiency can lead to a complex of local and systemic reactions aimed at eliminating the results of this influence. One of the environmentally significant chemical factors for the human organism is the element No. 27 of the Periodic System named Cobalt. Cobalt belongs to the group of microelements, i.e. is vital for the functioning of living organisms. However, in abundance like many other elements or more complex substances. It is toxic the first information on the toxicity of cobalt appeared as early as 1883, while at the same time it can itself act as antidote to cyanide intoxication (Afridi et al., 2011; Battaglia et al., 2009; Edel et al., 1994).

Sources of exposure to cobalt and inorganic cobalt compounds are both natural and anthropogenic (Fig. 1). Natural sources include wind-blown dust, seawater spray, volcanoes, forest fires, and continental and marine biogenic emissions. Anthropogenic sources include the burning 
of fossil fuels, sewage sludge, phosphate fertilizers, mining and smelting of cobalt ores, processing of cobalt alloys, and industries that use or process cobalt compounds (Czarnek et al., 2015).

Serious interest in the biochemistry of cobalt arose around 1934 due to severe diseases of cattle and sheep in various parts of the world (Russia, Scotland, Australia, New Zealand, Canada). Animals lost weight, appetite, became sluggish, anemic and eventually died (Edel et al., 1994). The presence of anemia suggested an iron deficiency. But it turned out that the matter is not in the iron itself, but in the presence of very small amounts of cobalt in the iron compounds (McKinney \& Rogers, 1992). Adding cobalt to the feed completely eliminated all toxic symptoms. Much less known is the fact that the active center of a number of enzymes contains cobalt, which is not included in $\mathrm{B}_{12}$. These are methylmalonyl-CoA-carboxytransferase, propionyl-CoA carboxylase. Cobalt can also act as a coenzyme in the composition of some pyrophosphatases, peptidases, arginases (Bruick, 2003). There is evidence that cobalt can affect the activity of enzymes, in particular, adenylate cyclase and several others (Edel et al., 1994). It has a particular effect on heme metabolism enzymes (Zadnipryany et al., 2017).

Cobalt is widely used in metallurgy, being a component of heavy metal alloys, also involved in diamond grinding, in the production of drying agents, pigments and catalysts (Battaglia et al., 2009; Czarnek et al., 2015). Cobalt radioisotopes are applied in industry, medicine, and nuclear research. In the air of the working area for the production of powdered cobalt, its concentration ranges from 0.675 to $10 \mathrm{mg} / \mathrm{m}^{3}$ (Edel et al., 1994), and in the production of heavy metal alloys area - from 14.6 to $37.4 \mathrm{mg} / \mathrm{m}^{3}$ (Goldoni et al., 2004). Common occupational diseases among the personnel of such industries are pneumonia, fibrosis and asthma (Malard et al., 2007). In acute rat experiment, a single 30-minute exposure of animals to a cobalt-containing aerosol (26-236 mg / $\left.\mathrm{m}^{3}\right)$ had resulted in lung congestion, edema, and hemorrhage (Patel et al., 2012). Cobalt can contribute to the development of tumors (Lison et al., 2001; Nemery et al., 1994), it is even listed as carcinogenic agents IARC (Agency for Research on Cancer of the International Health Organization), while its complex compounds have an antitumor effect.

So, the physiological and pathophysiological effects of cobalt are diverse. There is information about its influence on the metabolism of carbohydrates and lipids (Nemery et al., 1994), on the function of the nervous system and thyroid gland (Karovic et al., 2007), the state of the myocardium (Huy et al., 1973). It was the so-called "Beer cardiomyopathy" that drew attention to the toxic effects of cobalt, which led to the cessation of its use for the treatment of anemia. The fact is that in some countries for a number of years (the $60 \mathrm{~s}$ of the twentieth century), cobalt (1.2-1.5 mg / 1) was added to beer to improve foaming, and this led to serious diseases, including deaths among fans of this drink. A rather large number of old works, ranging from (Alexander, 1968; Ortega et al., 2009), indicate the

\section{Ways of Cobalt ions $\left(\mathrm{Co}^{2+}\right)$ into the human body}

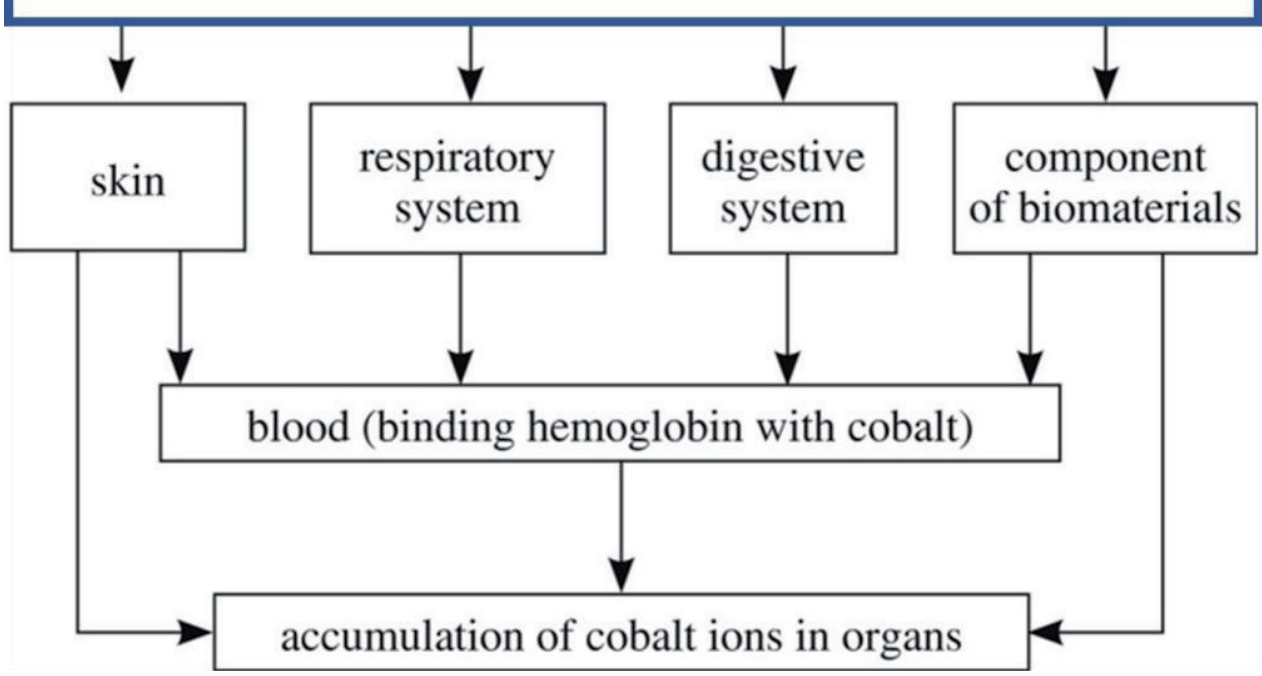

Figure 1. Ways of cobalt ions into body (Czarnek et al., 2015). The largest source of exposure to cobalt for the general population is the food supply. The estimated intake from food is 5-40 $\mu \mathrm{g} /$ day, most of which is inorganic cobalt 
effect of cobalt on blood pressure and vascular tone. The data are contradictory: both vasodilation and vasoconstriction effects were observed, both a decrease and an increase in pressure. Thus, the totality of experimental and epidemiological evidence is not sufficient to prove a role for cobalt in inducing toxic cardiomyopathies in cobalt-exposed industrial workers. However, in the context of this paper, the relationship between cobalt and cardiac hypoxia should be considered in more detail.

\section{Materials and methods}

Eighteen 3-6 months old mature male Wistar rats weighing 200-250 g were involved in the experiment. Cobalt chloride $\left(\mathrm{CoCl}_{2}\right)$ water solution was administered daily orally in low dosage ( $4 \mathrm{mg} / \mathrm{kg}$ ) which was considered to be cardiotoxic (Zadnipryany et al., 2017). Control intact group consisted of 6 rats. After 30 days of cobalt exposure the animals were sacrificed by decapitation under ether anaesthesia according to international standards and rules of animal bioethics.

The experiments were carried out according to the permission of the Academic Council of the Crimean Medical Institute (Act No. 103 of 30.11.77). The animal maintenance was approved by the Institutional Committee on Bioethics and is consistent with the Guidelines for the Care and Use of Laboratory Animals published by the US NIH (No. 85-23, revised in 1985).

Fragments of right and left ventricle after sampling was immediately placed in 4\% formaldehyde solution buffered to a $\mathrm{pH}$ of 7.2-7.4 with monosodium phosphate and processed through the usual technique for paraffin inclusion. For the immunohistochemical study, sections were cut using the same equipment, but with a thickness of $3 \mu \mathrm{m}$. Sections were collected on poly-Llysine coated slides, incubated in a thermostat at $37^{\circ} \mathrm{C}$ for 24 hours in order to obtain a perfect adhesion of the biological material to the surface of the histological slide, and then stained using antibodies Anti-vimentin Dako V9. After antigen retrieval, sections were cooled down to room temperature and were incubated for 30 minutes in a $1 \%$ hydrogen peroxide solution. The sections were next washed in PBS, followed by a blocking step of 30 minutes in $2 \%$ skim milk. Next, the slides were incubated with the primary antibodies overnight at $400 \mathrm{C}$, and the next day, the signal was amplified for 30 minutes using a peroxidase polymer based secondary detection system (EnVision, Dako).

The myocardial samples were sliced into 1- to 2-mm cubes and prepared for evaluation by transmission electron microscopy. The myocardium collected for fine structural evaluation was minced immediately into $0,5-\mathrm{mm}$ blocks and fixed by immersion in cold $2.5 \%$ glutaraldehyde with $0.1 \mathrm{M}$ sodium cacodylate buffered to $\mathrm{pH}$ 7.4. The cubes of myocardium were then postfixed in $1 \%$ osmium tetroxide in $0.1 \mathrm{M}$ cacodylate, processed, and embedded in Epon. Semithin sections were stained with tolouidine blue, and ultrathin sections, with lead citrate, and examined with a SELMI electron microscope.

Statistical processing of data was carried out using licensed software Microsoft Office Excel 2007 and Statistica 10.0 .

\section{Results}

Hypoxic effects of cobalt chloride were manifested as a significant decrease of body mass and heart mass of the exposed rats. According to measurements body mass had significantly lowered up to $19,49 \%$ and heart mass had diminished up to $11,76 \%$ compared with control values (Fig. 2). That can evidence the fact that cobalt may interfere the protein synthesis.

According to histological analysis cardiac lesions were found in all experimental rats. The investigation of myocardial tissue from the left ventricle by conventional histological staining, allowed us to observe that cobalt cardiomyopathy is characterized by significant changes within both cardiomyocytes as well as the interstitial connective tissue. Myocardial cells showed variable staining patterns due to changes in intracytoplasmic structures. The dystrophy and attenuation of the myofibrils was the most common observation. In some myocardial cells the reduction of myofibrils appeared relatively homogenous, translated into a reduction of staining intensity and attenuation of myofibrils, while in other cells the reduction of myofibrils was heterogeneous which resulted in a vacuolar sarcoplasmic pattern, particularly on cross-sections of myocardial cells (Fig. 3).

Most frequently myofibrils in the central area of the myocardial cells were less affected to those in the periphery, highlighting that the main cause of myofibril reduction could be a lower oxygen intake in the perinuclear area. The reduction of the contractile support of myocardial cells can explain the possible myocardial dysfunction that can lead to chronic heart failure. Nuclear changes were also quite common. Most often there were found deformed, twisted, hyperchromatic nuclei, with chromatin arranged in an uneven pattern; sometimes pyknotic nuclei with chromatin organized in homogeneous disintegrating blocks were seen. Nuclear changes were consistent with sarcoplasmic changes in the sense that if sarcoplasmic changes were significant, so were nuclear ones.

Myocardial connective tissue changes were sometimes significant and sometimes barely noticeable. The most frequent and constant change in this structure was the emergence and development of the initial fibrosis. In the early stages, this process was reported to be more intense in the 


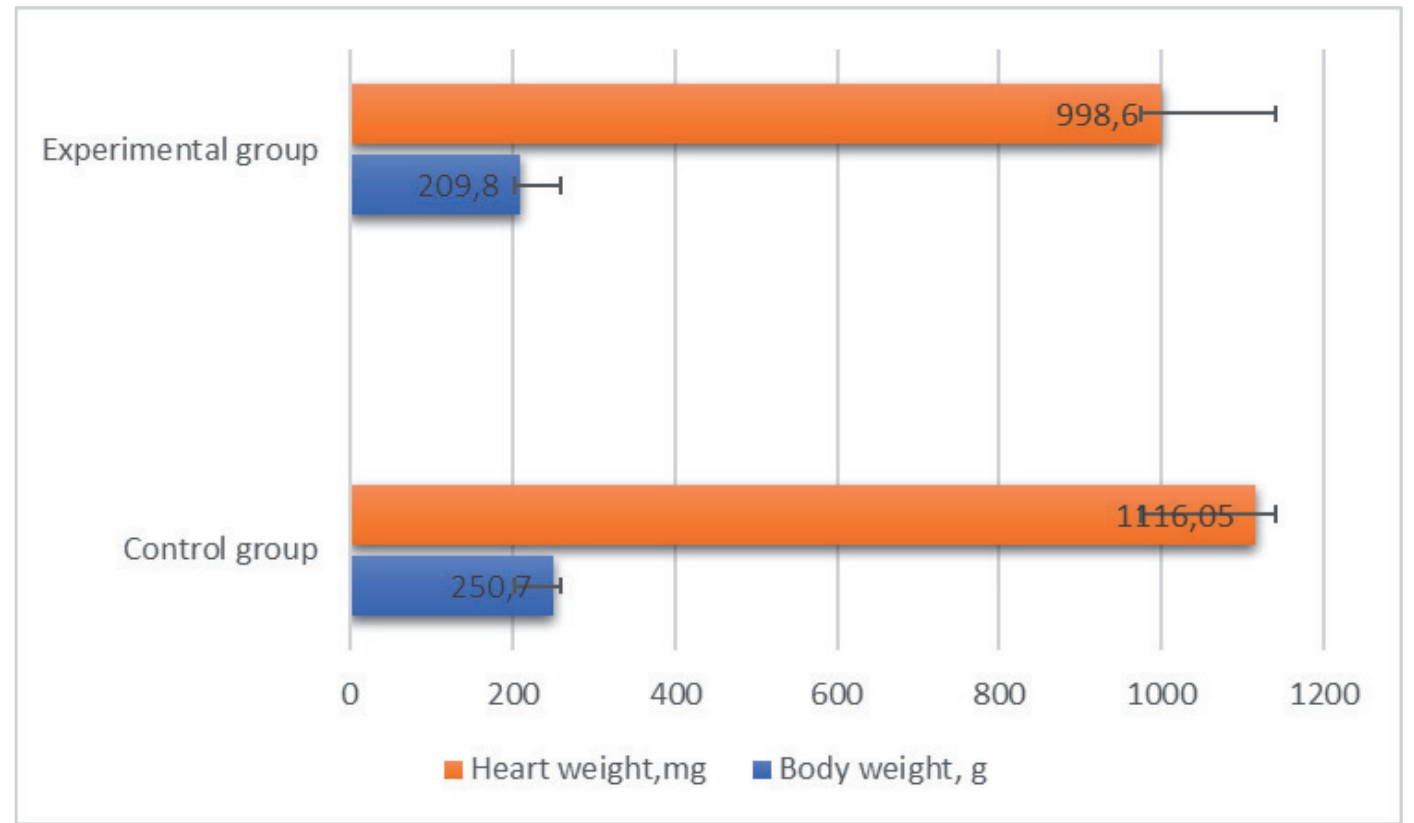

Figure 2. Body and heart weight in the control and experimental groups

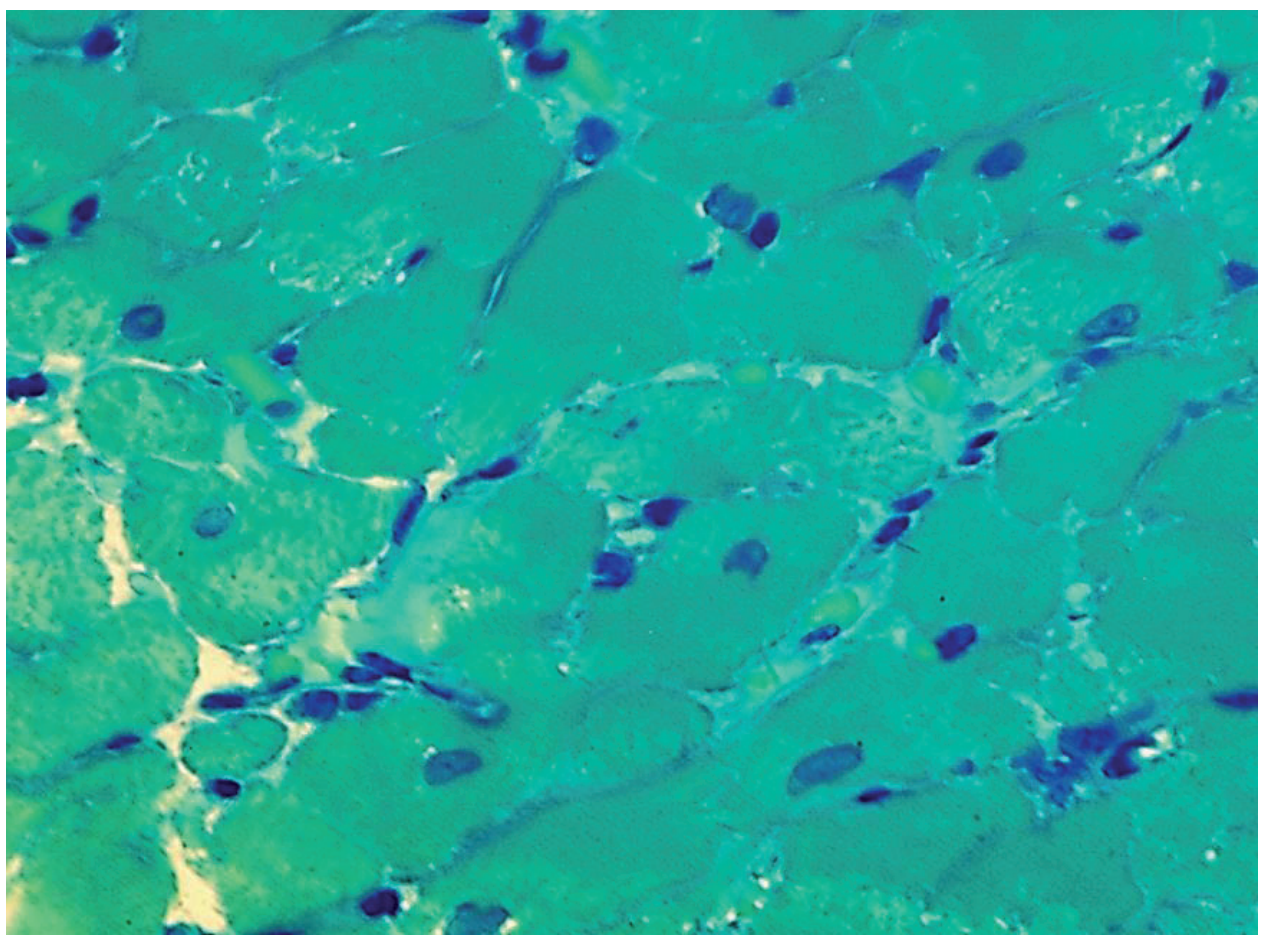

Figure 3. Pale perinuclear areas of cardiomyocytes represent swollen mitochondria. Epon embedded thin section, stained with toluidine blue $(\times 1000)$ 


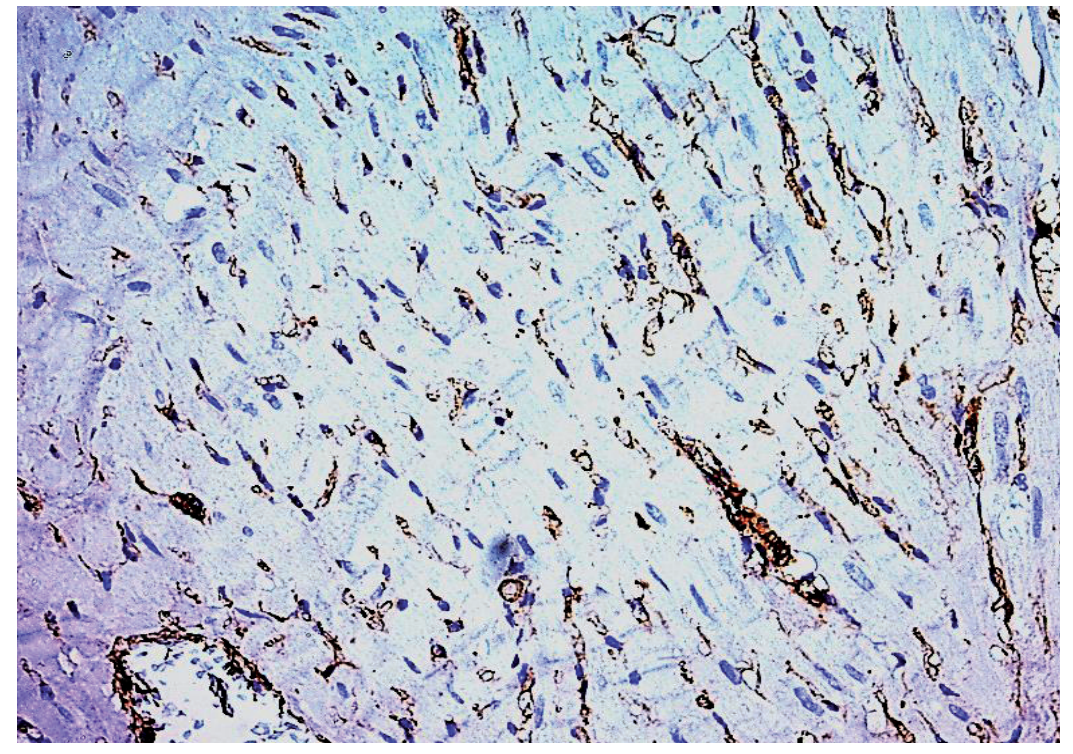

Figure 4. Intense reaction to vimentin (immunohistochemical staining for vimentin, $\times 200$ )

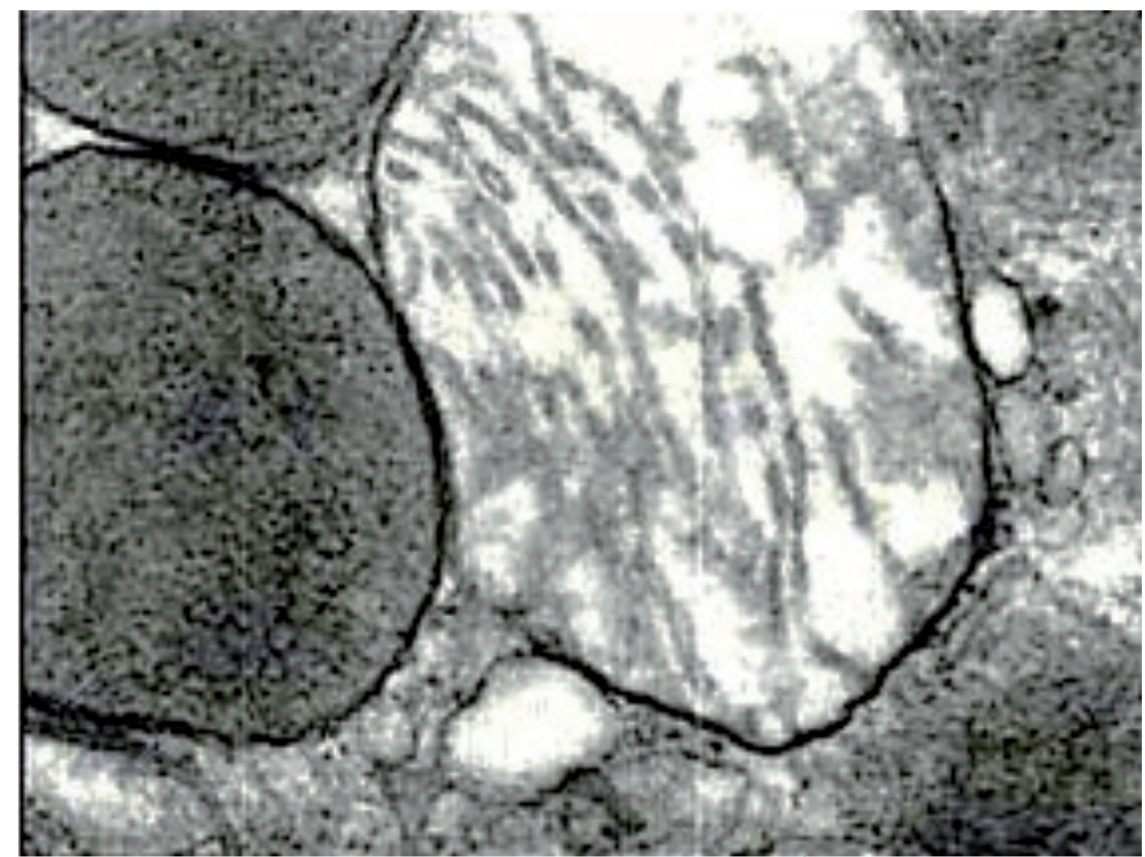

Figure 5. Electron microscopic presentation of swollen mitochondria and dilated sarcoplasmic reticulum (TEM x 20,000)

perivascular areas, which could be explained by the increasing synthesis activity of fibroblasts present in this space. At some level, the collagen synthesis expanded progressively, dissociating heart muscle fibers, isolating them from the neighboring ones. It is possible that interstitial fibrillogenic processes start due to chronic myocardial ischemia, which would explain cardiomyocyte suffering manifested by a reduction of intermediate filaments, cytoplasmic organelles and myofibrils.

For the evaluation of myocardial interstitial connective tissue reaction in dilated cardiomyopathy, we investigated the vimentin immunohistochemical reaction knowing that vimentin is a major intermediate filament protein present in mesenchymal-derived cells. In our study, we found that 
the reaction to vimentin was intense in the interstitial tissue of the ventricular myocardium, where proliferative and fibrotic processes were more intense (Fig. 4). We believe that vimentin specifically marked the fibroblast-type connective cells, responsible for collagen fibrillogenesis. In other words, the reaction to vimentin can be used for early diagnosis of cardiac sclerosis.

Ultrastructurally, the myocardium of control rats was normal. Myocardial lesions were seen in all cobalt-treated rats. Ultrastructural changes were more prominent in the left. Ultrastructural lesions consisted of swelling and disorganization of the mitochondria as evidenced by loss of inner membranes and cristae, leaving a distorted outer membrane (Fig. 5).

Plenty of mitochondria with disrupted inner and outer membranes had focal matrical densities (Fig. 5). The presence of dense osmophilic intramitochondrial particles can be pathognomonic pathological finding for cobalt-induced heart disease. Many cardiac cells, especially in the ventricles, contained swollen mitochondria that had a clear matrix and focal loss of cristae. Increased numbers of lipid droplets of varying sizes were observed throughout the myocardium. Some droplets contained increased peripheral electron densities especially in areas with pronounced mitochondrial changes.

The sarcoplasmic reticulum sometimes mainly between the myofilaments, but the sarcoplasmic reticulum appeared essentially normal in those fibers presenting minimal changes in the mitochondria and myofilaments. The my- ofibrillar changes, though observed in portions of the heart examined, were more frequent in the atria. Changes in the myofibrils varied from small localized areas of increased thickening of the Z-bands to loss of striations and early granular dissolution of the filaments. In some locations, short fragments of myofilaments containing Z-bands remained. These lesions were seen in areas where mitochondrial damage was more pronounced (Fig. 6).

\section{Discussion}

It should be noted that the numerous studies found in the available literature on the biological effect of cobalt can be divided into two large groups: the authors of one of them consider cobalt in an environmental aspect as a harmful natural agent and an industrial toxicant, without paying any attention at all to positive its effects on the body $(\mathrm{Pu}-$ lido et al., 2003), the authors of another group of works consider cobalt in the physiological aspect and focus on its erythropoietic function, in general, on mediated erythropoietin influence on blood formation, and recently - on its universal role in the activation of HIF, leaving out the general toxic manifestations of the biological action of element No. 27. In our work we tried to focus on the first aspect of the problem.

Long-term oral administration of cobalt resulted in diminished dietary intake and growth inhibition in the exposed rats compared to the controls. The harmful effects

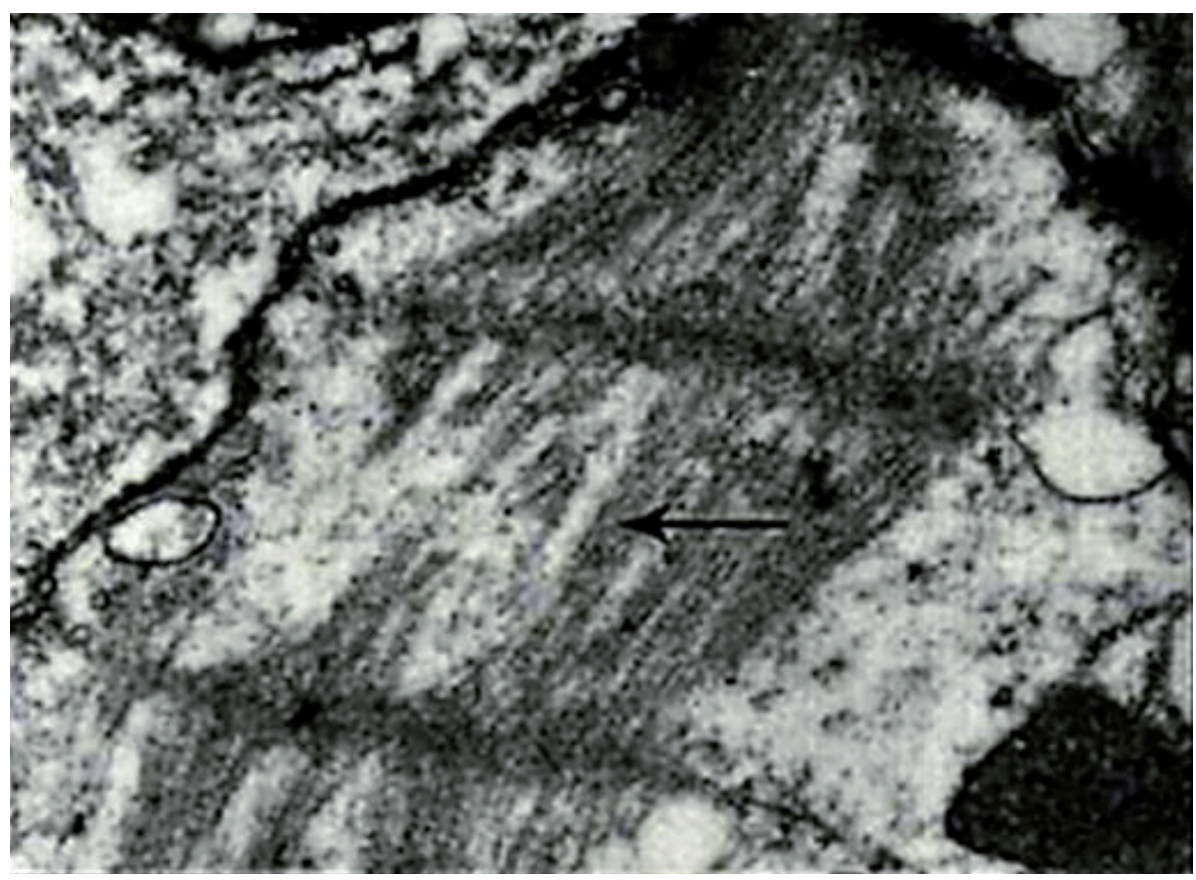

Figure 6. Severe disorientation of myofibrils with loss of Z-band material (arrow), (TEM x 10,000) 
of cobalt in high concentrations are attributed mainly and primarily to the fact that excessive intake of cobalt in the body is accompanied by a state of hypoxia or the cell's "feeling" of lack of oxygen (Simonsen et al., 2012). The basis of so many manifestations of the effect of cobalt on the functions of living organisms is one universal intracellular mechanism - activation of the so-called hypoxia-inducible factor, or HIF (hypoxia inducible factor) (Czarnek et al., 2015). This mechanism is not the only one. Recently, 85 genes have been identified in an experiment on the culture of human lung cells, the expression of which in response to exposure to cobalt is altered in high doses (Karovic et al., 2007). Among the proteins encoded by these genes are cobalt carrier proteins, suppressors and transcription factors of tumors, proteins produced in response to stress. Of the 85 proteins identified by the authors, only 7 were associated with HIF. Thus, the effects of increased cobalt doses on the body are not limited to imitation of hypoxia (Malard et al., 2007).

Myocardium from rats with experimental cobalt cardiomyopathy in our study revealed marked swollen mitochondria and moderate to severe structural changes in the sarcoplasmic reticulum and myofibrils. Electrondense particles were seen in some mitochondria. Previous electron microscopic studies of alcoholic cardiomyopathy revealed strikingly similar changes in the mitochondria in man. Hibbs et al. (1965) reported swollen mitochondria, loss of cristae, dense intramitochondrial inclusions, and other alterations in deformed mitochondria. In cobaltbeer-drinker's cardiomyopathy, Alexander (1968) examined biopsies from six men and noted the presence of electron-dense deposits in swollen mitochondria in one patient. Others observed a few mitochondria containing particulate inclusions (Alexander, 1968).

The reduced 'mitochondrial respiration' observed in the ultrastructural analysis may reflect an acute cobalt toxicity. Cobalt interferes with myocardial energy metabolism, as it irreversibly chelates with the dithiol form of lipoic acid, inactivating the coenzyme required for the oxidative decarboxylation of pyruvate to acetyl coenzyme A and alpha ketoglutarate to succinate. Pyruvate and lactate accumulation in the mitochondria increase osmotic pressure and results in water binding, oedema, and finally structural disruption. The dense osmiophilic particles observed in mitochondria have been described in rats as "pathognomonic" for cobalt toxicity (Battaglia et al., 2009).

Cobalt may interfere with the binding of calcium to the sarcolemma, the transport of calcium into the myocyte, and the inotropic effects of calcium. In addition, cobalt can interrupt the citric acid cycle and the generation of ATP by aerobic cellular respiration, inhibit the activity of respiratory chain enzymes and ATP production in mitochondria, and promote the generation of reactive oxygen species. This can result in a depression of cardiac function and changes in cardiac cell structure leading to the lipid dystrophy (Nemery et al., 1994).

The principal lesions observed in scattered myofilaments in male rats were areas of increased electron density and thickening of the Z-bands. Electron microscopic findings included separated myofibrils, Z-band degeneration, and loss of striations, increased amount of collagen fibers in the interstitial and perivascular space. That can cause an irreversible depression of cardiac systolic dysfunction and remodeling of myocardium.

\section{Conclusions}

Cobalt is widespread in the natural environment and can be formed as an effect of anthropogenic activity. Summarizing the in vivo experiments, it can be stated that the hematopoietic reaction that occurs at an early stage after the introduction of an excess amount of cobalt chloride into the body develops against the background of a severe hypoxic state of the whole organism, intoxication and heart deficiency in male rats. Electron microscopy showed characteristic vacuolar degeneration and loss of myofibrils, coiled and degenerated remnants of intercalated discs, swollen and distorted mitochondria with absent cristae with dense inclusions, and dilated sarcoplasmic reticulum. The reduction of the contractile support of myocardial cells can explain the possible myocardial dysfunction. Changes of the interstitial connective tissue were sometimes extensive and sometimes barely noticeable which can be predictable markers of myocardial remodeling.

Histological changes associated with dilated cardiomyopathy non-specific and not all may be present simultaneously. The fact that drug-induced heart disease, and in particular cobalt-induced cardiomyopathy, does not occur more often, as would be expected from the diversity of various mechanisms, is perhaps surprising. In spite of this, cardiotoxicity remains a major problem of hundreds of pharmaceutical agents, industrial chemicals and naturally occurring products and is often a limiting factor in treatment of certain diseases. Hence, it must be taken in account in the process of clinical decision making and treatment as well as in the process of drug research and development.

\section{References}

Afridi H.I., Kazi T.G., Kazi N., Kandhro G.A., Baig J.A., Jamali M.K., Arain M.B., Shah A.Q., Shah F., Khan S. \& Kolachi N.F., 2011, Association of environmental toxic elements in biological samples of myocardial infarction patients at different stages. Biol. Trace. Elem. Res. 141: 26-40. (PMID: 20480400 doi: 10.1007/ s12011-010-8713-2). 
Alexander C.S., 1968, The syndrome of cobalt-beer cardiomyopathy including ultrastructural changes on biopsy. J. Lab. Clin. Med. 72: 850.

Battaglia V., Compagnone A., Bandino A., Bragadin M., Rossi C.A., Zanetti F., Colombatto S., Grillo M.A. \& Toninello A., 2009, Cobalt induces oxidative stress in isolated liver mitochondria responsible for permeability transition and intrinsicapoptosis in hepatocyte primary cultures. Int. J. Biochem. Cell Biol. 41: 586-594. (PMID: 18708157 doi: 10.1016/j.biocel.2008.07.012).

Bruick R.K., 2003, Oxygen sensing in the hypoxic response pathway: regulation of the hypoxia-inducible transcription factor. Genes Dev. 17: 2614-2623. (PMID: 14597660 doi: 10.1101/gad.1145503).

Czarnek K., Terpiłowska S. \& Siwicki A.K., 2015, Selected aspects of the action of cobalt ions in the human body. Cent. Eur. J. Immunol. 40(2): 236-42. (PMID: 26557039 PMCID: PMC4637398 doi: 10.5114/ ceji.2015.52837).

Edel J., Pozzi G., Sabbioni E. Pietra R. \& Devos S., 1994, Metabolic and toxicological studies on cobalt. Sci. Total Environ. 150: 233-244.

Goldoni M., Catalani S., De Palma G. Manini P., Acampa O., Corradi M., Bergonzi R. Apostoli P. \& Mutti A., 2004, Exhaled breath condensate as a suitable matrix to assess lung dose and effects in workers exposed to cobalt and tungsten. Environ. Health Perspect. 112: 1293-1298. (PMID: 15345342 PMCID: PMC1247519 doi: 10.1289/ehp.7108).

Hibbs R.G., Black W.C., 1965, Electron microscopy of post-mortem changes in the rat myocardium. The Anatomical Record 147(2): 261-72. (PMID: 14065938 doi: 10.1002/ar.1091470209).

Huy N.D., Morin P.J., Mohiuddin S.M. \& Morin Y., 1973, Acute effects of cobalt on cardiac metabolism and mechanical performance. Can. J. Physiol. Pharmacol. 51: 46-51.

Karovic O., Tonazzini I., Robola N., Edstrцm E., Lцvdahl C., Fredholm B.B. \& Darŭ E., 2007, Toxic effects of cobalt in primary cultures of mouse astrocytes. Similarities with hypoxia and role of HIF-1alpha. Biochem Pharmacol. 73: 694-708. (PMID: 17169330 doi: 10.1016/j.bcp.2006.11.008).
Lison D., De Boeck M., Verougstraete V. \& Kirsch-Volders M., 2001, Update on the genotoxicity and carcinogenicity of cobalt compounds. Occup Environ Med. 58: 619625. PMID: 11555681 PMCID: PMC1740056

Malard V., Berenguer F., Pratt O. Ruat S., Steinmetz G. \& Quemeneur E., 2007, Global gene expression profiling in human lung cells exposed to cobalt. BMS Genomics. 8: 147-164. (PMID: 17553155 PMCID: PMC1904204 doi: 10.1186/1471-2164-8-147).

McKinney J. \& Rogers R., 1992, Metal bioavailability. Environ. Sci. Technol. 26(7): 1298-1299.

National Research Council, 1985, Guide for the Care and Use of Laboratory Animals, 6th ed. NIH Pub. No. 8623. Washington, DC: US Department of Health and Human Services.

Nemery B., Lewis C.P.L., \& Demedts M., 1994, Cobalt and possible oxidant-mediated toxicity. Sci. Total Environ. 150: 57-64.

Ortega R., Bresson C., Fraysse A., Sandre C., Devès G., Gombert C., Tabarant M., Bleuet P., Seznec H., Simionovici A., Moretto P., \& Moulin C., 2009, Cobalt distribution in keratinocyte cells indicates nuclear and perinuclear accumulation and interaction with magnesium and zinc homeostasis. Toxicol. Lett. 188:26-32. (PMID: 19433266 doi: 10.1016/j.toxlet.2009.02.024).

Patel E., Lynch Ch., Ruff V. \& Reynolds M., 2012, Coexposure to nickel and cobalt chloride enhances cytotoxicity and oxidative stress in human lung epithelial cells. Toxicol. Appl. Pharmacol. 258: 367-375. (PMID: 22172632 doi: 10.1016/j.taap.2011.11.019).

Pulido M.D. \& Parrish A.R., 2003, Metal-induced apoptosis: mechanisms. Mutat Res. 533: 227-241. (PMID: 14643423).

Simonsen L.O., Harbak H. \& Bennekou P., 2012, Cobalt metabolism and toxicology - a brief update. Sci Total Environ. 432: 210-215. (PMID: 22732165 doi: 10.1016/j.scitotenv.2012.06.009).

Zadnipryany I.V., Tretiakova O.S., Sataieva T.P. \& Zukow W., 2017, Experimental |review †of †cobalt †induced cardiomyopathy. Russian Open Medical Journal 6(1): 1-4. (http://www.romj.org/files/pdf/2017/romj-2017-0103. pdf). 